\title{
Rhodamine 6G as a Mediator of Silver Nanoparticles Aggregation
}

\author{
S. Kruszewski*, M. CYrankiewiCz \\ Medical Physics Division, Biophysics Department, Collegium Medicum of Nicolaus Copernicus University \\ ul. Jagiellońska 13, 85-067 Bydgoszcz, Poland
}

\begin{abstract}
Surface-enhanced Raman scattering is the phenomenon where a huge increase of Raman scattering intensity from molecules situated close to the metal nanoobjects is observed. Our study is focused on the method of SERSactivation of silver nanoparticles and, in the future, the application of thus obtained SERS substrates for biomedical purposes. As expected, the intensity of Raman scattering from rhodamine 6G used here as a SERS probe strongly increase during the early stages of aggregation of silver sol. Moreover, the evolution of extinction spectra and changes in the degree of the colloid aggregation observed in DLS measurements point out that molecules of the dye do not participate passively in the aggregation process but greatly affect its course.
\end{abstract}

DOI: 10.12693/APhysPolA.123.965

PACS: 33.20.Fb, 33.20.Kf, 61.46.Df, 78.67.Bf, 87.83.+a

\section{Introduction}

The recent great interest in noble metal nanoparticles is related to their ability to amplify the light scattered from molecules adsorbed on them. This optical phenomenon called surface-enhanced Raman scattering (SERS) was discovered and described in the 70's of 20th century $[1,2]$ but still remains the subject of intense reseArchiv. More recently SERS have also begun to be used as a microanalytical tool in biotechnological and biomedical study.

SERS is a combination of two mechanisms. The main contribution to the SERS originates from electromagnetic mechanism (EM) [3]. It is related to the resonance of surface plasmons localized on the metallic nanostructures that arises when the frequency of light matches the oscillation frequency of free conduction electrons of metal. Molecules adsorbed on the surface (or located in a close proximity) of nanoparticles are affected by electric field much stronger than in the absence of metal. The second contribution to the enhancement is a short-range resonant mechanism of chemical nature. This mechanism leads to the increase of molecular polarizability, mainly due to the formation of charge-transfer (CT) complexes between metal and adsorbate, and requires molecules to adsorb directly on the nanostructures, in so-called "active sites" [4].

The average intensity of Raman scattering from molecules dissolved in suspension containing dispersed isolated silver nanoparticles increases by a few orders of magnitude (typically about two orders) in comparison to pure solution (without silver nanoparticles) but this enhancement is still insufficient for practical SERS applications. The method to obtain significantly stronger SERS signal consists in partial aggregation of colloid [5]. It is

\footnotetext{
*corresponding author; e-mail: skrusz@cm.umk.pl
}

based on the fact that the extremely high electric field is induced in the junction between closely spaced nanoparticles, in so-called "hot-spots". The intensity of light Raman scattered by molecules occupying such places may rise up to 11-12 orders of magnitude [6]. This means that the total enhancement (the result of both mechanisms, EM and CT) makes possible the detection of SERS signal from a single molecule. Phenomenon of such giant enhancement was first observed in $1996[7,8]$.

The effect of chloride on the SERS-activation of silver colloid is well known [9]. Chloride ions reduce repulsive forces between nanoparticles and promote their aggregation. On the other hand, chloride can act like a catalyst in adsorption of various molecules on silver surface. The effect of various amount of chloride on the rate of aggregation (in the silver nanoparticles/chloride ions/SERS probe molecules system) has been the subject of earlier authors' works. The purpose of this paper is to find out what is the role of analyte (a compound used as SERS probe) in the aggregation of nanoparticles.

Rhodamine $6 \mathrm{G}$ is used in this work both as a SERS probe adsorbate and the aggregating agent. This cationic xanthene dye is widely used in industry (as a dye or gain medium in dye lasers) but also in biochemical and biomedical application, for example in fluorescence labeling. The most direct method that allows to examine the SERS-activity of silver nanoparticles $/ \mathrm{KCl} /$ rhodamine system is to record the Raman spectra of analyte. However, the analysis of SERS spectra can not give complete information about the mechanisms behind the observed enhancement, so in this work also other experimental techniques are used.

Studies presented in this paper are expected to contribute to the development of methods for obtaining small aggregates (composed of only a few nanoparticles) with SERS-active molecules trapped inside (in "hot spots") that will be preserved and protected from the environment by encapsulation in durable and biocompatible shells (a similar idea is presented in [10]). Therefore, understanding the mechanisms of adsorption of SERS 
probe molecules on the surface of the nanoparticles and their influence on the aggregation is particularly important. During further work, present authors intend to functionalize thus obtained systems with targeting ligands (antibodies) capable of recognizing and binding to specific receptors (antigens) on the surface of bacteria, viruses, or tumour cells. These systems can be perfect for Raman imaging and/or simultaneous detection of multiple antigen (by different functionalization of nanoparticles labeled with different SERS marker molecules).

\section{Experimental}

\subsection{Materials}

Silver nitrate $\left(\mathrm{AgNO}_{3}\right)$, trisodium citrate $\left(\mathrm{Na}_{3} \mathrm{C}_{6} \mathrm{H}_{5} \mathrm{O}_{7} .2 \mathrm{H}_{2} \mathrm{O}\right)$ and potassium chloride $(\mathrm{KCl})$ were obtained from POCh S.A. (Poland). Rhodamine $6 \mathrm{G}$ was purchased from Sigma-Aldrich. Mili-Q grade water $(18.2 \mathrm{M} \Omega \mathrm{m})$ was used for the preparation of all solutions.

\subsection{Silver colloids production and samples preparation}

The nanoparticles used in experiments were prepared using a slightly modified Lee and Meisel method [11]. For studies carried out in this work silver nanoparticles are obtained by mixing of $100 \mathrm{ml}$ of $1 \mathrm{mM}$ silver nitride with $2 \mathrm{ml}$ of $1 \%$ aqueous solution of trisodium citrate. The last solution was added in 4 portions. First portion $(0.2 \mathrm{ml})$ was added after silver nitride solution heating to temperature of $90^{\circ} \mathrm{C}$. Next, the solution was heated to about $96^{\circ} \mathrm{C}$ and after 20 minutes, the second portion equal $0.6 \mathrm{ml}$ was added. The next portions (3rd and $4 \mathrm{th}$ ) each equal $0.6 \mathrm{ml}$ were added after next 15 and 30 minutes, respectively. After adding the last portion, the temperature was still kept at $96{ }^{\circ} \mathrm{C}$ for about 30 minutes, and content of flask was occasionally stirred. Finally, the resultant mixture was cooled down to room temperature and replenished to the initial volume by addition of pure water. For further studies (i.e. SERS and extinction spectra measurements and particle sizing by DLS) it was 5 -fold diluted in water. The colloid obtained in such a way was stable and its extinction spectrum (as well as the SERS-efficiency) did not change for a long time (at least a few months). The stability of the colloid is the result of presence of a negatively charged citrate layer on the surface of nanoparticles [12].

$\mathrm{KCl}$ was used as the primary aggregating agent. All samples containing colloids with appropriate amount of this compound were shaken on vortex stirrer for $30 \mathrm{~s}$ after addition. Then the analyte (rhodamine 6G) was added and mixing was continued for next $30 \mathrm{~s}$. The thus prepared material was then transferred into three measuring cuvettes. The measurements were started immediately after the preparation of each sample.

\subsection{Instrumentation}

The absorbance spectra were collected using a twobeam V550 (Jasco Inc., USA) spectrophotometer in standard $10 \mathrm{~mm}$ path length optical glass cells. The time of single scan of extinction spectrum was about $140 \mathrm{~s}$.
The particle sizes were determined by photon correlation spectroscopy [13]. Fluctuations of the intensity of dynamically scattered light (DLS) were analyzed on the system composed of a photon counting unit PhotocorPC1 (based on Hamamatsu R6358-10 photomultiplier) and 128-chanells Photocor-FC correlator working in linear spacing mode. Both devices were purchased from Photocor Instruments Inc., USA. The power of the incident beam (632.8 nm light from a He-Ne laser source) was adjusted with circular variable neutral density filter (Thorlabs Inc., USA) and a width of the beam was reduced by using lenses and pinholes. Scattering angle was set to $90^{\circ}$. The laser light was polarized perpendicularly to the scattering plane. The single accumulation of scattered light took $15 \mathrm{~s}$ after which correlator was stopped, read, and restarted. The obtained autocorrelation functions were analyzed by means of originally developed Matlab scripts.

SERS spectra were recorded on DK480 (Spectral Products Inc., USA) $0.5 \mathrm{~m}$ spectrometer equipped with Atla U30-OE camera (Apogee Imaging Systems Inc., USA) with open-electrode front-illuminated CCD sensor and programmable thermoelectric cooler. Both the monochromator and the camera were controlled by KestrelSpec software (Catalina Scientific Corp., USA). The spectra were excited by $632.8 \mathrm{~nm}$ line of $35 \mathrm{~mW}$ He-Ne laser (Coherent Inc., USA). The acquisition time was $15 \mathrm{~s}$. To improve the precision of measurement, each recording of SERS spectrum was followed by $15 \mathrm{~s}$ accumulation of dark signal in order to perform appropriate background correction.

\section{Results and discussion}

\subsection{SERS spectra of rhodamine $6 G$ on silver nanoparticles}

In the spectrum of light scattered in SERS (and normal Raman as well [14]) process one can distinguish several narrow bands corresponding to the normal vibrations of the molecule. Fig. 1 shows series of spectra recorded for selected sample within the first hour after preparation.

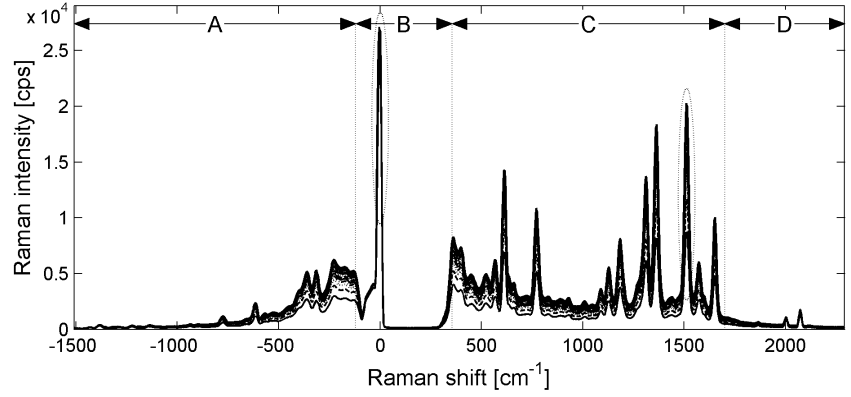

Fig. 1. Sample set of SERS spectra of rhodamine 6G on silver colloid recorded during 1-hour experiment. The four regions (A, B, C and D) are described in the text. Elastic scattering and Raman $\left(1510 \mathrm{~cm}^{-1}\right)$ bands are marked with ellipses. 
As it can be seen in Fig. 1, in the whole measurement range one can distinguish four areas different from each other. In the anti-Stokes area designated by "A", secondary lines of helium-neon laser overlap the relatively week Raman bands. In the range marked with "B", light reaching the detector is significantly attenuated by the Notch filter. In this region only elastically scattered He-Ne laser line $(632.81 \mathrm{~nm})$ emerges from the background. Spectral range with dominant rhodamine 6G Stokes bands is labeled by "C". It should be mentioned, that in this region the secondary laser lines are also present and their intensity sometimes can be comparable with the intensity of Raman bands (especially He-Ne line at about $1650 \mathrm{~cm}^{-1}$ ). In the last area marked with "D", only weaker laser lines can be distinguished.

In Fig. 1, the Mie-Rayleigh and the most prominent Raman band of rhodamine $6 \mathrm{G}$ at $1510 \mathrm{~cm}^{-1}$ (related to the motion of the xanthene ring) are enclosed with elongated ellipses. The intensity of these two lines will be used in the next section of this paper as the indicator of SERS efficiency during aggregation of silver nanoparticles.

\subsection{Time- and rhodamine $6 G$ concentration-dependence of colloid aggregation}

SERS experiment. As it was shown above, the intensity of Raman spectra of rhodamine $6 \mathrm{G}$ on silver colloid varies during experiment. This variation is the result of many factors. It mainly depends on the kinetics of analyte adsorption on the silver surface and on the progress of nanoparticle aggregation.

The plots in the Fig. 2 show some interesting dependencies. In the case of higher concentrations of rhodamine $6 \mathrm{G}$, the Raman signal drops monotonically starting already from the beginning of the measurement series, while for lower concentrations of dye the maximum of SERS intensity is reached only after a certain time. For rhodamine concentrations of $10 \mathrm{nM}$ and lower, even after an hour the measured signal continues to rise.

Similar trend is observed for the Mie and Rayleigh scattering. The maximum of intensity of elastically scattered light is achieved earlier for the sample containing higher amount of rhodamine. For the concentration of analyte higher than $0.1 \mu \mathrm{M}$, the maximum was reached already during recording of the first spectrum. This behavior can be interpreted as a consequence of rapid aggregation and (probably) partial sedimentation of colloid. The initial increase in the Mie-Rayleigh signal in the case of smaller concentration of dye can be related to formation of small aggregates that exhibit strong scattering near $633 \mathrm{~nm}$. Our numerical calculations prove that small assemblies of silver nanoparticles approximately equidistant from each other (1-2 nm) strongly scatter the light within the red range [15].

The increase of SERS signal of rhodamine 6G during the measurement series is the effect of the adsorption of dye on silver surface as well as the result of the formation of aggregates with "hot spots". Both processes do not run immediately and require some time. On the other hand,

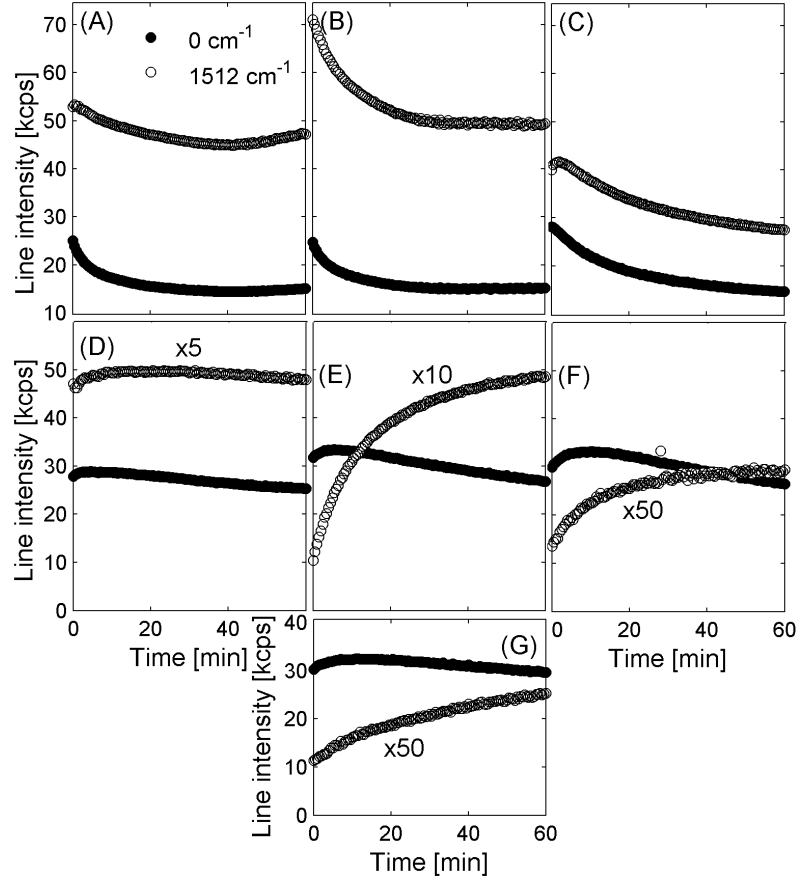

Fig. 2. The changes of the intensity of Mie and Rayleigh scattering (filled circles) and Raman Stokes scattering $1510 \mathrm{~cm}^{-1}$ (open circles) bands over time recorded for rhodamine $6 \mathrm{G}$ on silver sol with $25 \mathrm{mM}$ $\mathrm{KCl}$ at the following dye concentrations: (A) $1 \mu \mathrm{M}$, (B) $0.32 \mu \mathrm{M}$, (C) $0.1 \mu \mathrm{M}$, (D) $32 \mathrm{nM}$, (E) $10 \mathrm{nM}$, (F) $3.2 \mathrm{nM}$ and (G) $1 \mathrm{nM}$.

the averaged electric field around nanoparticle ensembles per one nanoparticle decreases with the size of aggregates (with the number of nanoparticles constituting the aggregates). Moreover, large aggregates become too heavy to float freely in a suspension and begin to fall to the bottom of a cuvette together with the dye molecules adsorbed on them.

The largest SERS intensity can be observed for sample with $0.3 \mu \mathrm{M}$ rhodamine. It can be assumed that this corresponds to the situation when the large part of analyte molecules was adsorbed on the surface of colloid aggregated to the extend at which a sufficient number of "hot-spots" was formed inside aggregates.

Analysis of extinction spectra. Processes occurring in the studied systems which determined their SERS-efficiency were also reflected in the evolution of the extinction spectra. The most important common feature of all spectra presented in Fig. 3 is a wide band (at $400-450 \mathrm{~nm}$ ) related to the dipole plasmon resonance of unaggregated nanoparticles. The second spectral feature is absorption band of rhodamine $6 \mathrm{G}$ at $527 \mathrm{~nm}$. The third one is broad band that arises in the red and near infrared region. The latter two features are observed only at higher concentration of rhodamine.

As one can see in Fig. 3, the extinction in violet-blue region significantly decreases as the concentration of the 


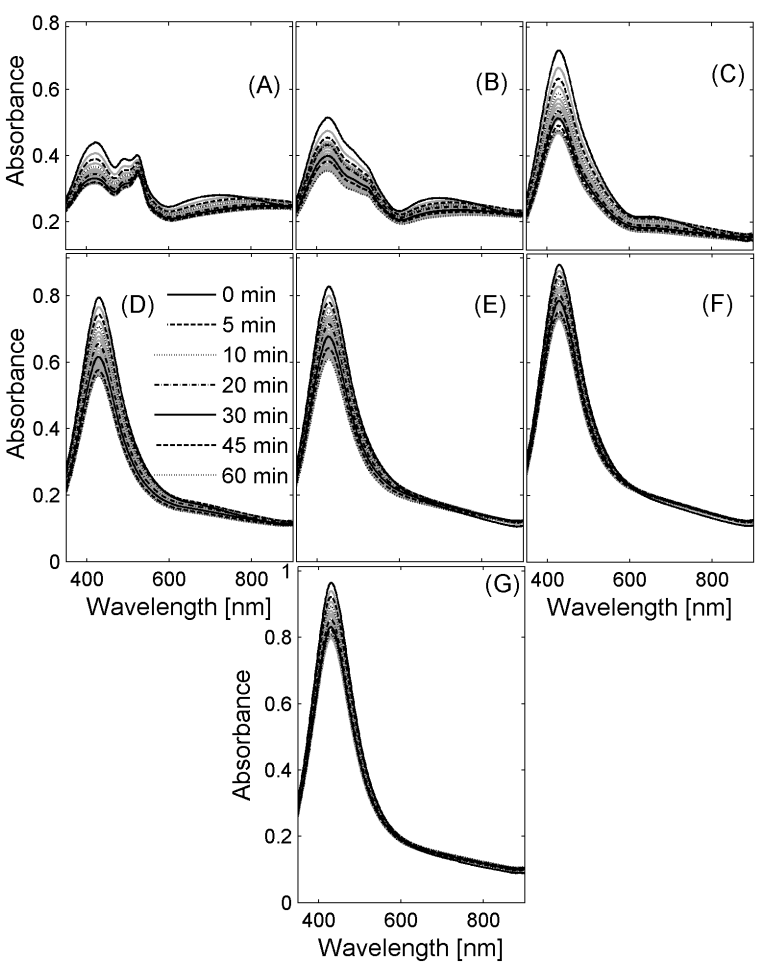

Fig. 3. The temporal evolution of extinction spectra of rhodamine $6 \mathrm{G}$ on silver sol with $25 \mathrm{mM} \mathrm{KCl}$ at the following dye concentrations: (A) $1 \mu \mathrm{M}$, (B) $0.32 \mu \mathrm{M}$, (C) $0.1 \mu \mathrm{M}$, (D) $32 \mathrm{nM}$, (E) $10 \mathrm{nM}$, (F) $3.2 \mathrm{nM}$ and (G) $1 \mathrm{nM}$.

dye rises that can be unambiguously interpreted as reduction in the number of isolated nanoparticles. An argument in support of this interpretation is the appearance of the second extinction band above $600 \mathrm{~nm}$ indicating (according to the theoretical predictions [16]) the formation of small assemblies of nanoparticles.

One can see that the extinction band related to the plasmon resonance of individual silver nanoparticles consistently decreases during 1-hour observation. The rate and extent of these changes strongly depend on the concentration of rhodamine $6 \mathrm{G}$. Their dynamics increases with the amount of the dye. One can also notice that the red band in extinction spectrum shifts to longer wavelengths with time. This shift is related to the increasing size of aggregates and further closing the distance between nanoparticles (see [15]).

Particle sizing by DLS. Fig. 4 shows the temporal changes of hydrodynamic radii of aggregates. These parameters ( $R_{H}$ or $R_{H 1}$ and $R_{H 2}$, respectively) were calculated on the basis of Stokes-Einstein equation [17] where translational diffusion coefficients were obtained from one- or two-exponential analysis of autocorrelation function of time-dependent fluctuations in the scattering intensity.

Considering the fact that the amount of rhodamine in all samples was many orders of magnitude lower than

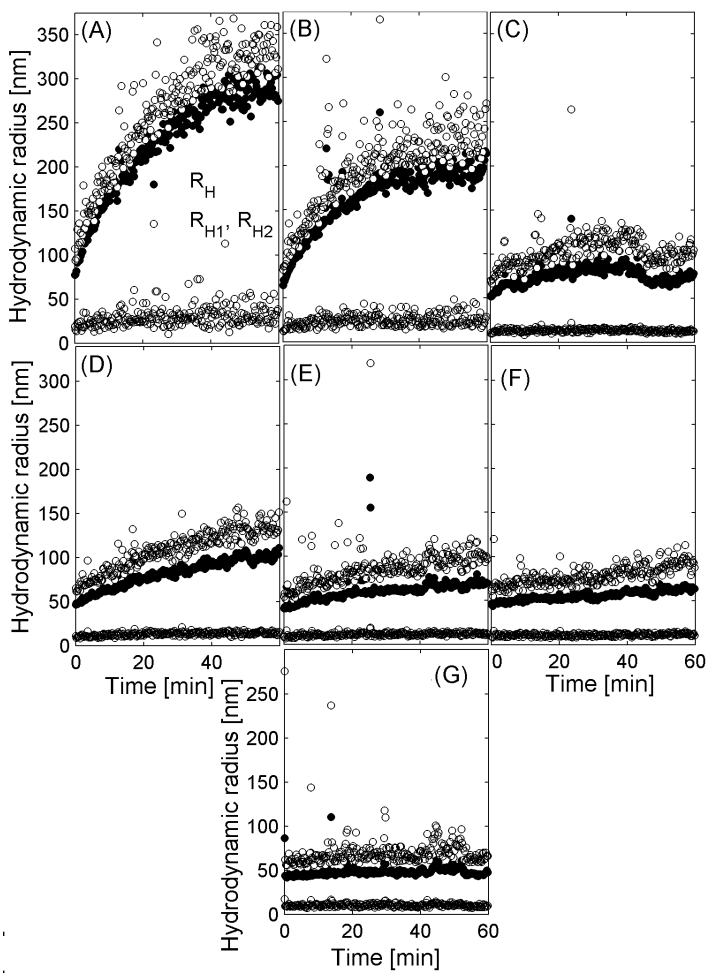

Fig. 4. The changes of hydrodynamic radii (the method of calculation is described in the text) of silver sol with $25 \mathrm{mM} \mathrm{KCl}$ and various amounts of rhodamine 6G: (A) $1 \mu \mathrm{M}$, (B) $0.32 \mu \mathrm{M}$, (C) $0.1 \mu \mathrm{M}$, (D) $32 \mathrm{nM},(\mathrm{E}) 10 \mathrm{nM}$, (F) $3.2 \mathrm{nM}$ and (G) $1 \mathrm{nM}$.

the amount of $\mathrm{KCl}$, one could expect that the influence of the dye on the aggregation process should be negligible. However, DLS measurements clearly show that the aggregation rate depends heavily on the concentration of rhodamine. The aggregation proceeds much faster for higher concentration of the dye. It seems that this effect should be smaller at lower concentrations of chlorides but further research work is necessary to confirm this supposition.

\subsection{The enhancement of Raman signal of rhodamine $6 G$ on silver sol}

Fig. 5 shows normal Raman spectrum of $0.2 \mathrm{mM}$ water solution of rhodamine $6 \mathrm{G}$ and the highest intensity SERS spectra of the $1 \mathrm{nM}$ and $10 \mathrm{nM}$ rhodamine $6 \mathrm{G}$ on silver colloid, respectively. The appearance of a disproportionately high peak at $1650 \mathrm{~cm}^{-1}$ is the result of overlapping Raman band and unfiltered laser line.

By measuring the (background subtracted) height of the chosen Raman band (the best choice seems to be a line at $1510 \mathrm{~cm}^{-1}$ ) in normal Raman and SERS spectra, one can determine the SERS enhancement factor. It can be calculated from the following formula:

$$
E F=\frac{I_{\mathrm{SERS}}}{I_{\mathrm{NR}}} \cdot \frac{C_{\mathrm{NR}}}{C_{\mathrm{SERS}}},
$$

where $C_{\text {SERS }}$ and $C_{\mathrm{NR}}$ are concentrations of analyte for SERS and normal Raman measurement, respectively, 


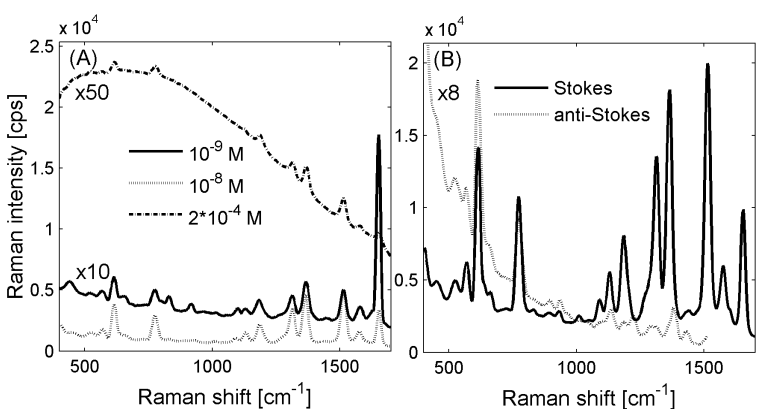

Fig. 5. The comparison of normal Raman spectrum of rhodamine $6 \mathrm{G}$ at concentration of $0.2 \mathrm{mM}$ and SERS spectra of the dye on silver colloid at concentrations of $1 \mathrm{nM}$ and $10 \mathrm{nM}$ (left panel). Stokes and anti-Stokes Raman bands shown in the common axis of Raman shift (right panel).

and $I_{\mathrm{SERS}}$ and $I_{\mathrm{NR}}$ are the corresponding intensities of selected vibrational bands. Usually, the intensity of vibrational bands is very week in comparison to fluorescence background but in the presented case (633 nm He-Ne laser line is far from the absorption/excitation maximum for rhodamine $6 \mathrm{G}$ ) it is relatively easy to state that maximum enhancement approaching a factor of about $3 \times 10^{7}$.

The observed SERS enhancement is, in fact, an average over all places occupied by molecules of rhodamine within the whole scattering volume. Locally (outside and inside aggregates) enhancement factor can vary from values near unity to values exceeding $10^{11}$. The high enhancement that was achieved in our experiments allowed the observation of anti-Stokes lines (see right panel of Fig. 5). Recording and analysis of the spectra in this range is the method to overcome completely the problem of unwanted fluorescence.

\section{Conclusions}

The fact that silver nanoparticles aggregate upon addition of chloride-containing compounds is well known and widely described in the relevant literature. However, there are no in-depth studies on the effect of rhodamine $6 \mathrm{G}$ on the rate of aggregation and the size of formed aggregates. Presented experiments confirm that potassium chloride is necessary (in presented case) to initiate the aggregation of silver nanoparticles. Moreover, the obtained results point out that the presence of rhodamine $6 \mathrm{G}$ definitely affects the flow of this process and this impact strongly depends on the concentration of dye.

Presented observations are qualitative only but, in the future, they certainly will be helpful to determine, control, and monitor the optimal conditions for preparation of SERS-active "nanocapsules".

\section{References}

[1] M. Fleishman, P.J. Hendra, A.J. McQuillan, Chem. Phys. Lett. 26, 163 (1974).

[2] D.L. Janmaire, R.P. Van Duyne, Electroanal. Chem. 84, 1 (1977).

[3] M. Moskovits, J. Raman Spectrosc. 36, 485 (2005).

[4] P. Hildebrandt, M. Stockburger, J. Phys. Chem. 88, 5935 (1984).

[5] H. Bengter, C. Tengroth, S.P. Jacobsson, J. Raman Spectrosc. 36, 1015 (2005).

[6] S. Kruszewski, M. Cyrankiewicz, Acta Physica Pol. A 121, A-68 (2012).

[7] K. Kneipp, Y. Wang, H. Kneipp, L.T. Perelman, I. Itzkan, R.R. Dasari, M.S. Feld, Phys. Rev. Lett. 78, 1667 (1997).

[8] S. Nie, S.R. Emory, Science 275, 1102 (1997).

[9] A. Otto, A. Bruckbauer, Y.X. Chen, J. Mol. Struct. 661-662, 501 (2003).

[10] G.B. Braun, S.J. Lee, T. Laurence, N. Fera, L. Fabris, G.C. Bazan, M. Moskovits, N.O. Reich, J. Phys. Chem. C 113, 13622 (2009).

[11] P. C. Lee, D. Meisel, J. Phys. Chem. 86, 3391 (1982).

[12] C.H. Munro, W.E. Smith, M. Garner, J. Clarkson, P.C. White, Langmuir 11, 3712 (1995).

[13] R. Pecora, Dynamic Light Scattering: Applications of Photon Correlation Spectroscopy, Plenum Press, New York, 1985.

[14] M. Połomska, L. Kubisz, R. Kalawski, G. Oszkinis, R. Filipiak, A. Mazurek, Acta Physica Pol. A 118, 136 (2010).

[15] S. Kruszewski, M. Cyrankiewicz, Acta Physica Pol. A 119, 1018 (2011).

[16] S.V. Karpov, A.L. Bas'ko, A.K. Popov, V.V. Slabko, Colloid J. 62, 699 (2000).

[17] L.H. Hanus, H.J. Ploehn, Langmuir 15, 3091 (1999). 